\title{
Nonactive Current Definition and Compensation Using a Shunt Active Filter
}

\author{
Yan Xu, Student Member, IEEE, John N. Chiasson, Senior Member, IEEE, Leon M. Tolbert, Senior Member, IEEE
}

\begin{abstract}
This paper presents a general definition of nonactive current/power and the implementation for a shunt compensation system. This definition is universal for different loads, such as non-periodic, unbalanced or single phase, and also flexible in terms of the compensation results. Unity power factor, pure sinusoidal source current, or zero non-active power supply from the utility can be achieved according to different compensation requirements. In addition, the corresponding current rating and energy storage requirements of the compensation system are also presented.
\end{abstract}

Index Terms-active filter, reactive power, non-active power, shunt compensator, non-periodic current.

\section{INTRODUCTION}

$\mathrm{N}_{\mathrm{e}}$ onlinear loads such as arc furnaces and power electronics converters continue to be added to the power power grid at an increasing rate. They draw highly distorted currents from the utility as well as cause distortion of the voltages. Most power electronics converters generate harmonic components whose frequencies are integer multiples of the system fundamental frequency. However, in some cases, such as cycloconverters, the line currents may contain both sub-harmonics (frequency lower than fundamental frequency) and super-harmonics (frequency higher than fundamental frequency but not an integer multiple of it). These waveforms are considered as nonperiodic although mathematically the currents may still have a periodic waveform. In any event, the period of these currents is not equal to the period of the fundamental voltage [1], [2]. An arc furnace is another example of a non-linear load that generates non-periodic currents because it draws rapidly changing power from the source and the wave shape and amplitude are constantly changing. Voltage flicker and harmonic penetration problems associated with arc furnaces have been reported in several papers [3]-[5].

A transient disturbance may also be considered as one kind of non-periodic current from the compensation point of view. The disturbance may be caused by the sudden addition of a large load to the system such as starting a motor, a fault, etc.

Y. Xu, J. N. Chiasson, and L. M. Tolbert, are with the Department of Electrical and Computer Engineering, The University of Tennessee, Knoxville, TN 37996-2100 USA (e-mail: yxu3@utk.edu, chiasson@utk.edu, tolbert@utk.edu).
The definition and compensation of non-sinusoidal and non-periodic currents and power has been a source of concern for some time [6]-[11]. However, most of the previous efforts have focused on the compensation of periodic nonsinusoidal currents instead of non-periodic currents. The diversity of the features of non-periodic currents makes their compensation quite difficult, and theoretically, their compensation is very different than that for periodic distorted currents. However, from a practical point of view, in both cases a sine wave with a constant rms magnitude is preferred for the source current after compensation. Some previous papers by the authors [11], [12] elaborate a new definition of non-active power/current.

This definition is applied to a compensation system using a conventional shunt active filter. This compensator must inject all of the non-active components that are the difference between the desired source currents and the required load currents. Based on this compensation system, several factors related to the definition itself and the implementation are discussed. These factors include the averaging interval $T_{C}$, the coupling inductance $L_{C}$, the DC link voltage $v_{d c}$, and the capacitance requirement $C$ of the shunt compensator. They are determined by the characteristics of the load, the rating limits of the compensator, and the desired compensation results.

\section{Definition of Non-Active CURRENT/Power}

Instantaneous active power is defined as the time rate of energy generation, transfer, or utilization, where $M$ is the phase number of the system:

$$
p(t)=\sum_{i=1}^{M} p_{i}(t)=\sum_{i=1}^{M} v_{i}(t) i_{i}(t) .
$$

The nonactive current/power definitions are based on this definition and an extension of Fryze's idea of nonactive current/power [1]. The definitions of instantaneous active current $i_{p}(t)$ and nonactive current $i_{q}(t)$ are:

$$
\begin{aligned}
& i_{p}(t)=\frac{P_{L}(t)}{V_{p}^{2}(t)} v_{p}(t) \\
& i_{q}(t)=i(t)-i_{p}(t)
\end{aligned}
$$




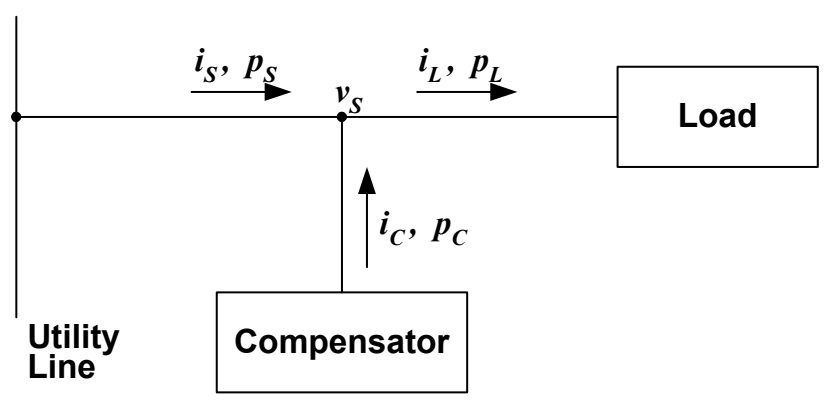

Fig. 1. A shunt compensator configuration.

where $P_{L}(t)$ is the average power of $p(t)$ over the interval $\left[t-T_{C}, t\right]:$

$$
P_{L}(t)=\frac{1}{T c} \int_{t-T c}^{t} p(\tau) d \tau,
$$

and $v_{p}(t)$ and $V_{p}(t)$ are the instantaneous and rms values of the reference voltage:

$$
V_{P}(t)=\sqrt{\frac{1}{T_{C}} \int_{t-T_{C}}^{t} v_{P}^{2}(\tau) d \tau} .
$$

The definitions are applied to a shunt compensation system as shown in Fig. 1. The load current $i_{l}(t)$ is divided into $i_{p}(t)$ and $i_{q}(t)$, according to the definitions above. The compensator provides the compensation current $i_{c}(t)=i_{q}(t)$, so that the utility need only to provide the source current $i_{s}(t)=i_{p}(t)$. Let

$$
P_{X}(t)=\frac{1}{T_{C}} \int_{t-T_{C}}^{t} p_{X}(\tau) d \tau=\frac{1}{T_{C}} \int_{t-T_{C}}^{t} v_{S}(\tau) i_{X}(\tau) d \tau,
$$

where $\mathrm{X}=S, L$ or $C$, so $P_{S}(t)$ and $P_{C}(t)$ are the active power and the nonactive power, respectively. Then

$$
P_{S}(t)+P_{C}(t)=P_{L}(t),
$$

and

$$
P_{S}(t)=P_{L}(t), P_{C}(t)=0 \text { as } t \rightarrow \infty .
$$

The shunt compensator consists only of passive components (inductor and/or capacitor) and/or switching devices. Consequently, no external power source is needed because it provides non-active power only. Here the compensator's power loss is neglected.

In (3) and (4), $T_{C}$ is the averaging interval. Theoretically, $T_{C}$ can be chosen as any arbitrary value in the case of nonperiodic currents. However, it is desirable for the interval to be an integer multiple of the line frequency period because of the desire that the source current be sinusoidal and have the same frequency as the source voltage frequency. In general, the period of the line voltage is not the same as the period of the quasi-periodic current $i_{p}(t)$. Thus, different $T_{C}$ will result in quite different source and compensator currents. $T_{C}$ can be zero, one fundamental cycle, one-half cycle, or multiple cycles, depending on the compensation objectives and the passive components' energy storage capacity.

It was shown in [12] that this new definition has the following features: 1) flexible to meet different compensation objectives; 2) valid for non-sinusoidal and non-periodic systems; 3) valid for single phase and poly-phase systems. Table I illustrates that by choosing different voltage reference and time averaging intervals, different source currents will result. Because of its flexibility in regards to compensation objectives, this definition is quite suitable for analyzing the compensation of non-periodic currents. This will be shown in Section IV.

TABLE I. PARAMETERS FOR DIFFERENT COMPENSATION OBJECTIVES

\begin{tabular}{|l|c|c|l|}
\hline Compensation Objective & $v_{\boldsymbol{P}}$ & $\boldsymbol{T}_{\boldsymbol{C}}$ & Resulting Source Current \\
\hline $\begin{array}{l}\text { Single-phase or polyphase } \\
\text { reactive current }\end{array}$ & $v$ & $T / 2$ or $T$ & $\begin{array}{l}\text { Unity pf and sinusoidal for } \\
\text { sinusoidal } v_{s}\end{array}$ \\
\hline $\begin{array}{l}\text { Single-phase or polyphase } \\
\text { reactive current and } \\
\text { harmonic current }\end{array}$ & $v_{f}$ & $T / 2$ or $T$ & $\begin{array}{l}\text { Unity pf and sinusoidal } \\
\text { regardless of } v_{s} \text { distortion }\end{array}$ \\
\hline $\begin{array}{l}\text { Instantaneous reactive } \\
\text { power for polyphase system }\end{array}$ & $v$ & $T_{C} \rightarrow 0$ & $\begin{array}{l}\text { Instantaneously unity pf for } \\
\text { polyphase system }\end{array}$ \\
\hline $\begin{array}{l}\text { Non-periodic disturbance } \\
\text { current }\end{array}$ & $v_{f}$ & $n T$ & $\begin{array}{l}\text { Reduced amplitude and near } \\
\text { sine wave with unity pf }\end{array}$ \\
\hline $\begin{array}{l}\text { Subharmonic current } \\
\text { Substic }\end{array}$ & $v_{f}$ & $n T$ & $\begin{array}{l}\text { Pure sine wave or smoothed } \\
\text { sine wave with unity pf }\end{array}$ \\
\hline $\begin{array}{l}\text { Stochastic non-periodic } \\
\text { current }\end{array}$ & $v_{f}$ & $n T$ & $\begin{array}{l}\text { Smoothed sine wave with } \\
\text { unity pf }\end{array}$ \\
\hline
\end{tabular}

\section{SYSTEM IMPLEMENTATION AND CONTROL}

This definition is independent of the shunt compensator system, i.e., the power electronic based compensator could be a normal inverter or a multilevel inverter; and the control strategy could be PWM or others. The definition gives the reference current that the compensator needs to generate, according to the system voltage, load current, compensation requirement, and the rating limits of the compensation system. In this paper, a PWM inverter is used.

As shown in Fig. 2,

$$
L_{c} \frac{d i_{c}}{d t}=v_{c}-v_{s} .
$$

Therefore, (9) is the mathematical representation of the feedback controller, and the control block diagram is shown in Fig. 3.

$$
v_{c}^{*}=\frac{s K_{P}+K_{I}}{s}\left(i_{c}^{*}-i_{c}\right)+v_{s} .
$$

The system voltage $v_{S}$ and load current $i_{L}$ are measured and input to the non-active current calculation block, in which the 


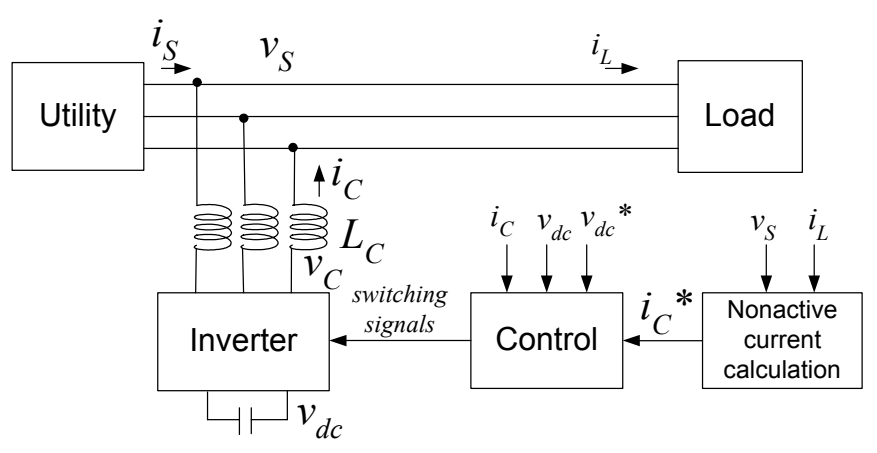

Fig. 2. System Implementation.

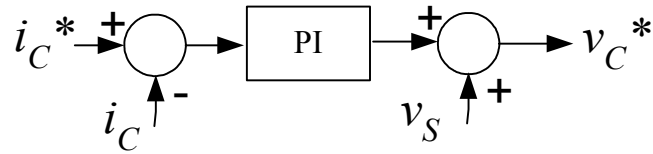

Fig. 3. Block diagram of the controller.

reference of the non-active current that the compensator needs to generate is calculated according to the definition presented in the previous section. In the control block, the DC link voltage is regulated and the compensator output voltage $v_{C}$ is calculated. The PWM pulse signals are then used to generate the control waveform $v_{C}$. The connecting inductance $L_{C}$ between the system and the compensator filters the ripple in the output current of the compensator and provides the mechanism to control $i_{C}$ by specifying $v_{C}$.

\section{NON-ACTIVE POWER COMPENSATION}

The compensation system has been simulated using Simulink/MatLab. The definition is valid for single-phase or multiphase, sinusoidal or non-sinusoidal, periodic or nonperiodic, balanced or unbalanced system. Four different loads are simulated, and the results are shown in this section.

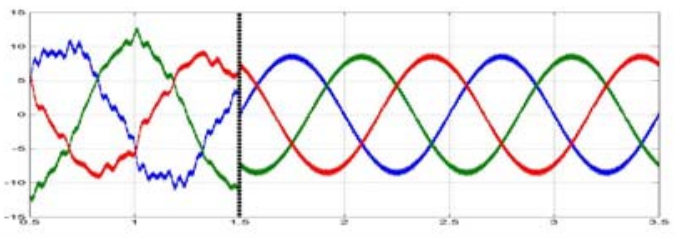

(a) Source current $i_{S}$ before and after compensation

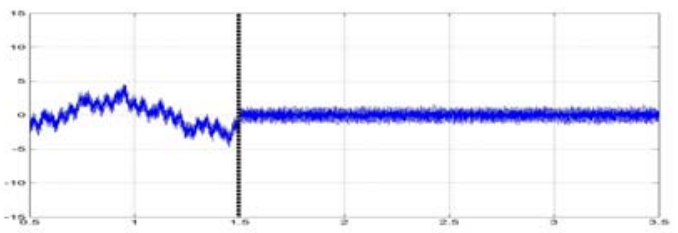

(b) Neutral current $i_{N}$ before and after compensation

Fig. 4. Simulation of harmonics unbalanced current compensation.

\section{A. Three-phase Harmonic Unbalanced Load}

In this case, load current has harmonics and is not balanced in phase $a, b$, and $c$, as shown in Fig. 4. The waveforms to the left of the dashed lines are source current and neutral current before compensation, which is actually the same as the load current. The source current is distorted by harmonics, and the neutral current is not zero.

For compensation of periodic currents with fundamental period $T$, choosing different $T_{C}$ alone does not change the source current characteristics. With reference to (3) and (4), the rms value of a periodic quantity does not depend on the time averaging interval $T_{C}$ if it is an integer multiple of $T / 2$.

So, with $T_{C}=T / 2$, the source current and neutral current after compensation are shown on the right side of Fig. 4a and $4 \mathrm{~b}$. The non-active component in the load current is completely compensated, and the source current now is purely sinusoidal, in phase with the system voltage, and balanced.

\section{B. Three-phase Rectifier Load}

A three-phase rectifier is a typical non-linear load. The phase $a$ current of a diode-based rectifier is shown in Fig. 5a. Basically, it is a periodic waveform which can be decomposed into a Fourier's series as in the previous case. However, the rectifier current is not continuous, and the variation of the current with time (di/dt) of the non-active current is very large (approaching infinity), as shown in Fig. $5 \mathrm{c}$. Therefore, there are special issues in the compensation of a rectifier load.

In the compensation of harmonic loads in the previous

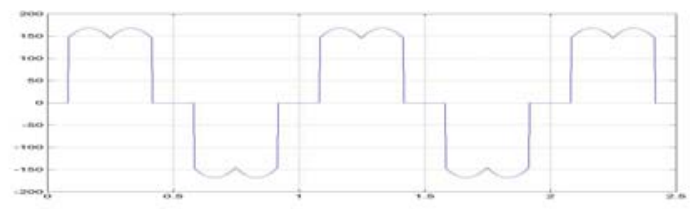

(a) Load current phase $a i_{L a}$.

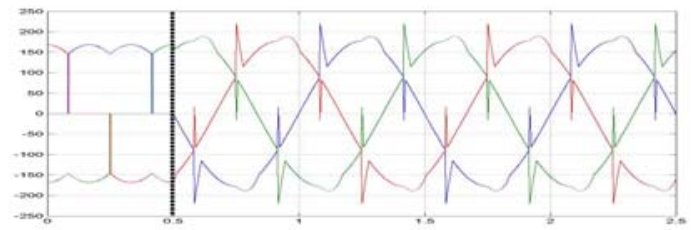

(b) Source current $i_{S}$ before and after compensation.

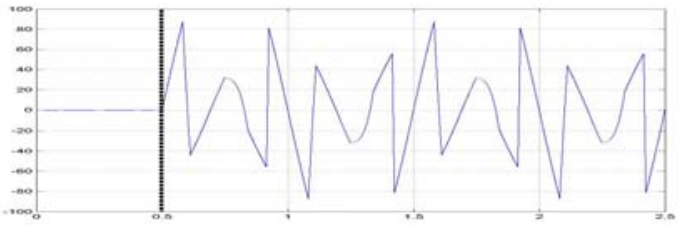

(c) Compensation current phase $a i_{C a}$.

Fig. 5. Simulation of rectifier load compensation. 


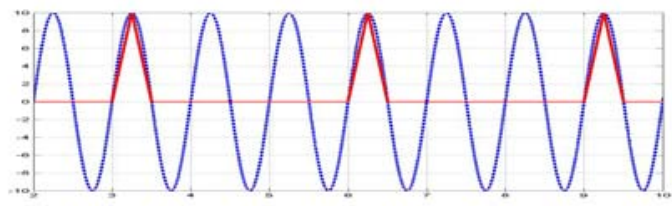

(a) voltage phase $a v_{S a}$ and load current phase $a i_{L a}$.

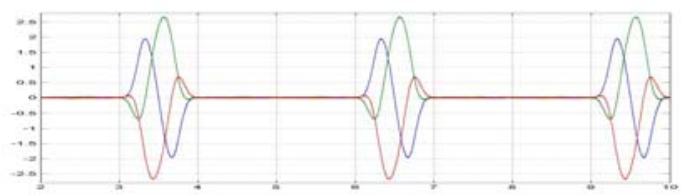

(b) Source current $i_{S}\left(T_{C}=T / 2\right)$.

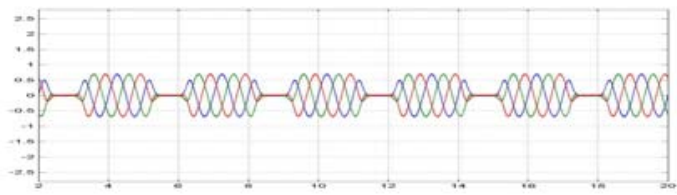

(c) Source current $i_{S}\left(T_{C}=2 T\right)$.

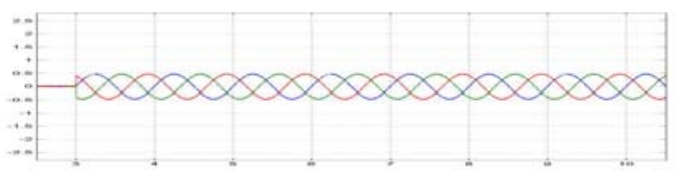

(d) Source current $i_{S}\left(T_{C}=3 T\right)$.

Fig. 6. Simulation of single-phase pulse load compensation.

subsection, the amplitude of $v_{C}$ is about the same as $v_{S}$, and a DC link voltage $v_{d c}$ having a value equal to the peak value of $v_{S}$ will be enough to fulfill the compensation task. However, in rectifier load compensation, the high di/dt demands high $v_{C}$, hence $v_{d c}$ must be much greater than $v_{S}$. In this simulation, $v_{d c}=2 v_{S}$, and there are significant overshoots in source current (see Fig. 5b). These overshoots are due to the high $d i / d t$ and to a relatively small value of $v_{d c}$. Increasing $v_{d c}$ can mitigate the overshoot, but also causes higher power rating and current rating of the compensator, i.e., a more expensive inverter.

\section{Single-phase Pulse Load}

If a single-phase load is connected to the three-phase utility, the single-phase current drawn from the system causes the unbalance of the system. Non-sinusoidal load current, or individual high amplitude pulse load current also introduces high non-active components to the system.

In this case, the load current is a single-phase pulse current, with a period of $3 T$ ( $T$ is the fundamental period of the voltage). The positive pulse load current (red) occurs every 3 cycles and has a triangular shape as shown in Fig. 6a together with sinusoidal system voltage $v_{S}$ (blue).

In this case, the averaging interval $T_{C}$ is a critical factor to the compensation. Figs. $6 \mathrm{~b}, 6 \mathrm{c}$, and $6 \mathrm{~d}$ show the three-phase source currents after compensation where $T_{C}$ is $T / 2,2 T$, and $3 T$, respectively. With the increasing of $\left.T_{C}, 1\right) i_{S}$ is closer to a sine wave; 2) the three phases are more balanced; 3$) i_{S}$ is

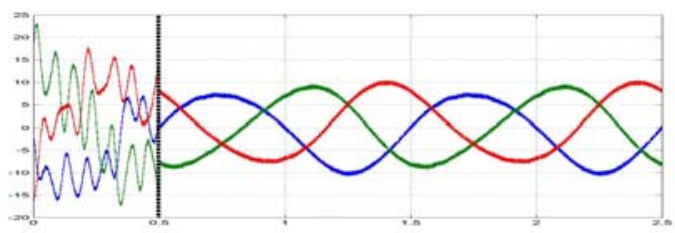

(a) Source current $i_{S}\left(T_{C}=T / 2\right)$.

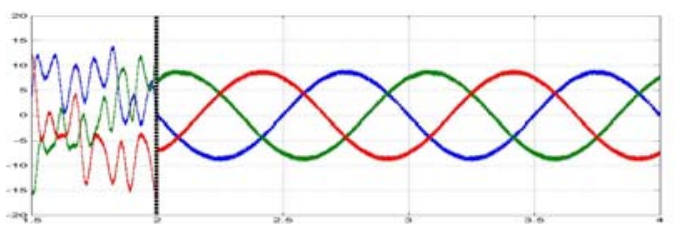

(b) Source current $i_{S}\left(T_{C}=2 T\right)$.

Fig. 7. Simulation of stochastic load compensation.

always in phase with the voltage; 4) the magnitude of $i_{S}$ is decreasing. However, by increasing $T_{C}$, the compensator current $i_{C}$ is also increasing, and consequently, the capacitance rating and switching current rating must increase as well. Depending on the load characteristics, compensator requirements, and the compensation results desired, a $T_{C}$ can usually be chosen to meet all the objectives. In this case for example, $T_{C}=2 T$ has a good source current without too high of a compensator rating.

\section{Stochastic Load}

A stochastic load is non-periodic, or theoretically, the period $T$ is infinity (a period much larger than the fundamental period of the utility). In the latter case, there are subharmonics in the load, whose frequencies are not an integer multiple of the fundamental frequency. The nonactive components in a stochastic load cannot be completely compensated by choosing $T_{C}$ as $T / 2$ or $T$, or even several times $T$.

In Fig. 7a, the waveforms to the left of the dashed line are the source currents before compensation (i.e., load currents), and the right part are the source currents after compensation, with $T_{C}=T / 2$. There is still significant non-active component in $i_{S}$, with variable peak values and non-sinusoidal waveform shape. In Fig. $7 \mathrm{~b}, i_{S}$ is closer to a sine wave with less nonactive component. Here a longer $T_{C}$ "smooths" the source current waveforms. Theoretically, $i_{S}$ could be a pure sine wave if $T_{C}$ goes to infinity, but in practice, such a $T_{C}$ cannot be implemented nor is it necessary. If $T_{C}$ is large enough, increasing $T_{C}$ further will not typically improve the compensation results significantly. For example, in this case shown in Fig. 7, the total harmonic distortion (THD) of $i_{S}$ is $4 \%$ with $T_{C}=T / 2$, and when $T_{C}=T / 2$, the THD is only slightly smaller than $4 \%$. So there is no need to increase $T_{C}$ to a larger value as the small decrease in THD is not worth the larger cost (higher ratings of the compensator and higher expenses). 


\section{DISCUSSION}

\section{A. Averaging Interval, $T_{C}$}

If there are only harmonics in the load current, as in Section IV.A, $T_{C}$ does not change the compensation results as long as it is an integral multiple of $T / 2$, where $T$ is the fundamental period of the system. Here the non-active current is completely compensated and a purely sinusoidal, unity PF source current is achieved.

However, in other cases, such as in parts B, C, and D of Section IV, $T_{C}$ has significant influence on the compensation results, and the power and energy storage rating of the compensator's components. With longer $T_{C}$, a better source current will result, but at the cost of higher power rating for the switches and capacitance. There is a tradeoff between better compensation and higher system ratings (i.e., cost). On the other hand, a longer $T_{C}$ does not necessarily yield a better source current waveform. For a specific system, there is an appropriate $T_{C}$ with which the compensation can be achieved. For example, in part $\mathrm{C}$ of Section IV, the pulse load $T_{C}$ depends on the period of the pulses.

\section{B. Coupling Inductance, $L_{C}$}

The inductance $L_{C}$ between the power system and the compensator could be the inductance of a step-up transformer or a coupling reactor. It acts as the filter of the compensation current $i_{C}$, which has high ripple content.

If $L_{C}$ is too small, it cannot filter the ripple in the compensation current $i_{C}$; and if $L_{C}$ is too large, the timeconstant of the system will be so large that $i_{C}$ cannot track the reference, which results in inadequate operation of the compensation system.

$L_{C}$ is inversely proportional to the rms value of load current when the ripple in the source current is limited to a specific percentage (e.g. $5 \%$ ):

$$
L_{c}=K / I_{L}, \quad \text { where } K \text { is a constant. }
$$

\section{DC Link Voltage, $V_{d c}$}

Rewrite (8) here:

$$
L_{c} \frac{d i_{c}}{d t}=v_{c}-v_{s} .
$$

For a given $L_{C}$, the change in current with time $d i_{C} / d t$ is determined by the voltage difference between $v_{C}$ and $v_{S}$, so a fast current change in $i_{C}$ requires a large $v_{C}$, which in turn requires a high voltage for the DC link of the inverter. Usually it is enough to have the DC link voltage higher than the peak value of the system voltage, but for some applications like the rectifier load and the pulse load in Section IV, the DC link voltage is 3-5 times the system voltage to mitigate the overshoot of the source current caused by the non-active current not being able to track the reference closely enough.

\section{DC Link Capacitance Rating $C$}

According to the definition in (7), the average power of the compensator $P_{C}(t)$ over $T_{C}$ is zero. However, the instantaneous power is not necessarily zero. The compensator generally has a capacitor for energy storage, and this capacitor operates in two modes: charge and discharge. Different capacitance values are required to fulfill different compensation tasks. The maximum energy stored in the capacitor is at the time $t_{\max }$ when the capacitor goes from charge to discharge or vice versa. The minimum energy is zero at the time $t_{\min }$ when $v_{C}=0$, so that the maximum stored energy is

$$
\Delta E=\int_{t_{\min }}^{t_{\max }} v^{T} i_{q} d t
$$

The capacitance required of the compensator is a function of the stored energy and voltage rating as $\mathrm{C}=2 \Delta \mathrm{E} /\left(\mathrm{V}_{\mathrm{dc}}\right)^{2}$.

\section{CONCLUSIONS}

A new definition of non-active power/current was presented. It has been applied to a shunt non-active power compensation system, and the implementation of the system was simulated. The simulation results of several different cases indicate that the definition is viable for different systems including single-phase or poly-phase, sinusoidal or non-sinusoidal, periodic or non-periodic, balanced or unbalanced systems. The definition is flexible in that it is applicable regardless of the configuration or the control strategies of the compensation system.

\section{ACKNOWLEDGEMENT}

We would like to thank the National Science Foundation for supporting this work through contract NSF ECS-0093884.

\section{REFERENCES}

[1] H. Akagi, Y. Kanazawa, A. Nabae, "Instantaneous Reactive Power Compensators Comprising Switching Devices without Energy Storage Components," IEEE Trans. Ind. Appl., vol. 20, May/June 1984, pp. 625-630.

[2] L. Rossetto, P. Tenti, "Evaluation of Instantaneous Power Terms in Multi-Phase Systems: Techniques and Application to Power Conditioning Equipment," ETEP, vol. 4, no. 6, Nov./Dec. 1994.

[3] F. Z. Peng, J. S. Lai, "Reactive Power and Harmonic Compensation Based on the Generalized Instantaneous Reactive Power Theory for Three-Phase Power Systems," Proceedings of the 7th International Conference on Harmonics and Quality of Power, Las Vegas, NV, October 16-18, 1996, pp. 83-89.

[4] L. M. Tolbert, T. G. Habetler, "Survey of Active and Non-Active Power Definition," IEEE International Power Electronics Congress, October 15-19, 2000, Acapulco, Mexico, pp. 73-79.

[5] F. Z. Peng, L. M. Tolbert, Z. Qian, "Definitions and Compensation of Non-Active Current in Power Systems," IEEE Power Electronics Specialists Conference, Cairns, Australia, June 23-27, 2002, pp. 17791784. 
[6] G. Carpinelli, M. Di Manno, al., "AC and DC Arc Furnaces: A Comparison on Some Power Quality Aspects," IEEE Power Engineering Society Summer Meeting, 1999, pp. 499-506.

[7] H. Akagi, "Active filters and Energy Storage Systems Operated under Non-periodic Conditions," IEEE Power Engineering Society Summer Meeting, Seattle, Washington, July 15-20, 2000, pp. 965-970.

[8] E. H. Watanabe, M. Aredes, "Compensation of Non-Periodic Currents Using the Instantaneous Power Theory," IEEE Power Engineering Society Summer Meeting, Seattle, Washington, July 15-20, 2000, pp. 994-999.

[9] L. S. Czarnecki, "Non-Periodic Currents: Their Properties, Identification and Compensation Fundamentals," IEEE Power Engineering Society Summer Meeting, Seattle, Washington, July 15-20, 2000, pp. 971-976.

[10] S. R. Mendis, M. T. Bishop, J. F. Witte, "Investigations of Voltage Flicker in Electric Arc Furnace Power Systems," IEEE Industry Applications Magazine, vol. 2, no. 1, Jan./Feb. 1996, pp. 28-34.

[11] Y. Xu, L. M. Tolbert, F. Z. Peng, J. N. Chiasson, J. Chen, "Compensation-based Non-active Power Definition," IEEE Power Electronics Letters, vol. 1, no. 2, June 2003, pp. 45-50.

[12] L. M. Tolbert, Y. Xu, J. Chen, F. Z. Peng, J. N. Chiasson, "Compensation of Irregular Currents with Active Filters," IEEE Power Engineering Society General Meeting, Toronto, Canada, July 13-18, 2003, pp. 1278-1283.

\section{BIOGRAPHIES}

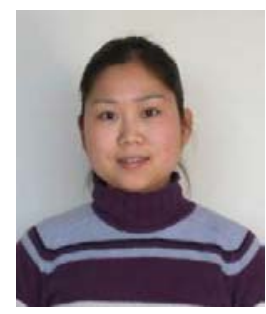

Yan Xu (S 2002) received the B.E. in Electric Power Engineering from Shanghai Jiaotong University, Shanghai, China and M.S. in Electric Power Engineering from North China Electric Power University, Beijing, China.

She worked in Anhui Electric Power Company Ma'anshan Branch, China from 1995 to 1998. She was an engineer in the Power Dispatch Department. She worked on the SCADA system and contributed to several SCADA projects in the distributions and dispatch center.

She presently is a Ph.D. student in Electrical Engineering at The University of Tennessee. Her research interests include non-active power compensation and pricing, and its application in distributed energy systems.

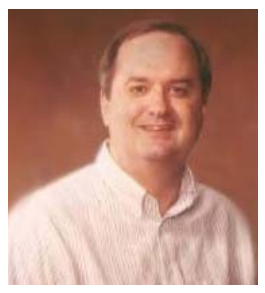

John N. Chiasson (S 1982 - M 1984 - SM 2003) received his Bachelor's in Mathematics from the University of Arizona, his M.S. in Electrical Engineering from Washington State University, and his $\mathrm{Ph} . \mathrm{D}$. in Controls from the University of Minnesota.

His work in industry started at Boeing Aerospace from 1978 to 1979 in the area of flight controls, guidance and navigation. From 1982-1983, he worked at Control Data in the area of CAD systems, and from 1984-1985 he worked at Honeywell Science and Technology Center in the area of inertial navigation. His latest stint in industry was from 1996-1999 at ABB DaimlerBenz Transportation where he worked in the development of AC motor propulsion systems, real-time simulators, and the stability analysis of $\mathrm{AC}$ propulsion systems. Since 1999, he has been on the faculty of Electrical and Computer Engineering at The University of Tennessee. He does research in the areas of the control of electric motor drives, multilevel converters, hybrid electric vehicles as well as mathematical systems theory.

Dr. Chiasson is an associate editor of the IEEE Transactions on Control Systems Technology.

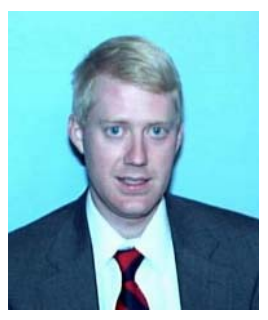

Leon M. Tolbert (S 1989 - M 1991 - SM 1998) received the B.E.E., M.S., and Ph.D. in Electrical Engineering from the Georgia Institute of Technology, Atlanta, Georgia

$\mathrm{He}$ joined the Engineering Division of Lockheed Martin Energy Systems in 1991 and worked on several electrical distribution projects at the three U.S. Department of Energy plants in Oak Ridge, TN. In 1997, he became a research engineer in the Power Electronics and Electric Machinery Research Center at the Oak Ridge National Laboratory. In 1999, he was appointed as an assistant professor in the Department of Electrical and Computer Engineering at the University of Tennessee, Knoxville. He is an adjunct participant at the Oak Ridge National Laboratory and conducts joint research at the National Transportation Research Center (NTRC). He does research in the areas of electric power conversion for distributed energy sources, motor drives, multilevel converters, hybrid electric vehicles, and application of $\mathrm{SiC}$ power electronics.

Dr. Tolbert is a registered Professional Engineer in the state of Tennessee. $\mathrm{He}$ is the recipient of a National Science Foundation CAREER Award and the 2001 IEEE Industry Applications Society Outstanding Young Member Award. He is an associate editor of the IEEE Power Electronics Letters. 